\section{Avances en el conocimiento de la relación entre la movilidad activa a la escuela y el entorno urbano}

\author{
Carla Hermida \\ ORCID: https://orcid.org/0000-0002-1095-7215 \\ Universidad del Azuay, Cuenca, Ecuador. \\ Correo electrónico: chermida@uazuay.edu.ec
}

\author{
Gabriela Naranjo \\ ORCID: https://orcid.org/0000-0003-0570-7446 \\ Pontificia Universidad Católica del Ecuador, \\ Quito, Ecuador.
}

\author{
Jaime Peña \\ ORCID: https://orcid.org/0000-0002-1231-7721 \\ Universidad del Azuay, Cuenca, Ecuador.
}

\begin{abstract}
Resumen
Se ha demostrado que la movilidad activa para los niños en edad escolar es fundamental para la salud, las relaciones sociales y el desempeño académico. Por ello, resulta preocupante que, en las últimas décadas, la movilidad activa hacia las escuelas haya disminuido. A pesar de que existe un creciente interés en promover la movilidad escolar a pie y en bicicleta, la evidencia científica es dispersa y poco estructurada, lo que dificulta la toma de decisiones adecuadas. En este artículo buscamos aportar a la sistematización del conocimiento acerca de movilidad escolar activa a través de una revisión de la literatura científica de los años 2009-2019 incluida en la base de datos bibliográfica Scopus. Se evidencia que las decisiones al respecto son tomadas principalmente por los padres, quienes ven como una barrera fundamental para la movilidad activa la inseguridad, tanto vial como personal; sin embargo, también inciden factores relacionados con el entorno construido, las condiciones sociodemográficas de los barrios, la distancia, el clima y la conveniencia de los padres. La revisión de la literatura evidencia también la concentración de este tipo de estudios en países desarrollados, y la incipiente investigación sobre la temática en países en vías de desarrollo.
\end{abstract}

Palabras clave

Movilidad activa, movilidad escolar, movilidad no motorizada, transporte sustentable

\section{Advances in the Knowledge of the Relationship between Active Mobility to School and the Urban Environment}

\author{
Adriana Quezada \\ ORCID: http://orcid.org/0000-0002-2335-8422 \\ Universidad de Cuenca, Cuenca, Ecuador.
}

\author{
Daniel Orellana \\ ORCID: http://orcid.org/0000-0001-8945-2035 \\ Universidad de Cuenca, Cuenca, Ecuador.
}

Financiamiento: El artículo fue desarrollado con el apoyo del Concurso CEPRA XIII, Código: CEPRA-2019-05 "Rutas escolares seguras". Institución: CEDIA. La Convocatoria permanente de proyectos de investigación, Código: INV-PCAR-A001-CEPRAXIII-2019-05, Universidad del Azuay, y el Proyecto: 2019_012_ CEDIA, Universidad de Cuenca.

\begin{abstract}
Active mobility for school-age children has been demonstrated to be critical to health, social relationships, and academic performance. That is why it is worrying that active mobility to schools has decreased in recent decades. Despite the growing interest in promoting school mobility on foot and by bicycle, the scientific evidence is scattered and poorly structured, making it difficult to make good decisions. This article aims to contribute to the systematization of knowledge about active school mobility through a review of scientific literature of the years 2009-2019, included in the bibliographic database Scopus. Parents make decisions about mobility mainly by parents, who see insecurity and road safety as barriers to active mobility. Factors related to the built environment, the socio-demographic conditions of the neighborhoods, the distance, the climate, and the convenience of the parents also have an influence. The literature review has also evidenced the concentration of studies in developed countries and the developing research on the subject in developing countries.
\end{abstract}

\section{Keywords}

Active mobility, non-motorized mobility, school mobility, sustainable transportation 


\section{Introducción}

Se ha comprobado que la movilidad activa a la escuela genera impactos positivos en los niños, ya que contribuye en diferentes aspectos: 1 ) mejora el estado físico (Mitra, 2013); 2) incide en el desarrollo de aptitudes para la interacción social, en el mejor rendimiento en los estudios y en el desarrollo de la cognición espacial (Mokkink et al., 2010); y 3) contribuye a mejorar las condiciones de los entornos mediante la reducción de emisiones de gas de efecto invernadero, la contaminación del aire y la pacificación de las calles (Goodman et al., 2019). Sin embargo, varios estudios demuestran que la movilidad activa a la escuela (a pie o en bicicleta) ha disminuido sensiblemente en las últimas décadas (Davison et al., 2008; Rothman et al., 2018). Esta tendencia incide directamente en los beneficios que la población infantil y la comunidad en general, pueden obtener de la práctica de estos modos de transporte.

En este artículo presentamos un trabajo que se basó en la búsqueda de artículos científicos en la base de datos de Scopus, para lo cual se identificaron y utilizaron diferentes combinaciones de las palabras clave en inglés: "school" "active mobility", "active commuting", "independent mobility", "walking", "cycling", "child". La búsqueda se realizó durante los meses de septiembre y octubre de 2019. Se restringió a las siguientes condiciones: 1) los artículos debían estar enfocados en la movilidad activa de niños en edad escolar (desde una mirada de su movilidad cotidiana, no como una práctica deportiva); 2) los artículos debían describir metodologías para la identificación de factores que inciden en la movilidad activa de escolares; 3 ) se seleccionaron artículos entre 2009-2019, con excepción de la revisión de literatura de Davison et al. (2008) que permitió entender el contexto anterior a la última década.

Luego de un proceso de selección, primero por título y luego por resumen, finalmente, se revisaron 27 artículos científicos (Tabla 1), los cuales se sistematizan en este documento.

El artículo se ha organizado de la siguiente manera: se inicia con un reporte y descripción de los enfoques que se han utilizado para estudiar los factores que afectan las decisiones sobre la movilidad activa a la escuela (Tabla 2) y, en segundo lugar, por unidades de observación: ya sean niños y niñas en edad escolar (en adelante "escolares"), o sus padres, madres o representantes (en adelante "padres y/o madres") (Tabla 3). Posteriormente, se recogen los factores prevalentes identificados que inciden en la decisión de caminar o ir en bicicleta a la escuela (Tabla 4); se empieza con una sección exclusiva para la seguridad, dada su incidencia en las decisiones en torno a la movilidad activa; y luego se exponen los factores restantes: entorno construido, las condiciones sociodemográficas de los barrios, la distancia, el clima y la conveniencia de los padres y/ o madres.

\section{Marco teórico}

Han sido varios los programas implementados en diferentes ciudades para motivar la movilidad activa a las escuelas (Teixeira et al., 2019; Villa-González et al., 2018). Por lo general, estos se basan en procesos de preparación, promoción, implementación y planteamiento de políticas, e incluyen tanto a la escuela, como a los padres y a la comunidad. En los 18 países de la Unión Europea, por ejemplo, se promueve el programa Traffic Snake Game, que consiste en dar un reconocimiento a los niños cada vez que alcanzan cierto número de viajes sustentables; de acuerdo con los resultados presentados, entre los años 2014 y 2017, el proyecto logró incrementar los viajes sustentables, no solo durante la campaña, sino una vez finalizada esta. Así también, el programa School Streets in Bolzano (Italia), implementado entre junio de 2017 y mayo de 2020, consistió en una suma de acciones tales como: contar con guardias que ayudan a los niños a cruzar las intersecciones, un pedibus y el cierre temporal de las calles aledañas a las escuelas.

En Estados Unidos, el Safe Routes to School Program consiste en diversos programas de motivación, capacitación e iniciativas de mejoramiento de la infraestructura. 
De acuerdo con McDonald et al. (2014), de las 801 escuelas que implementaron el programa durante cinco años, todas demostraron un incremento promedio del $43 \%$ en la movilización hacia ellas, ya sea caminando o en bicicleta; de este porcentaje, el 25 $\%$ se relaciona con los programas de educación y motivación y el $18 \%$ con las mejoras que se realizaron en infraestructura. En algunos países como Canadá, Estados Unidos, Australia, Nueva Zelanda y otros de Europa se ha implementado el Walking School Bus, un proyecto en el que los padres realizan turnos para recoger a varios niños en la ruta a la escuela a pie (Teixeira et al., 2019). De acuerdo con los hallazgos de Jones et al. (2019), este programa es uno de los más exitosos en los Estados Unidos cuando se lo compara con otras iniciativas; al respecto, Smith. et al. (2015) concluyen que es exitoso por incrementar, tanto la caminata como una opción de transporte, como los niveles de actividad en los niños.

Por otro lado, a nivel mundial, y principalmente en países desarrollados, varios grupos de investigación han enfocado sus esfuerzos en el desarrollo de metodologías para el estudio de la movilidad activa en los niños de edad escolar. Grupos de investigación como Active Living Research (Estados Unidos), Healthy Active Living and Obesity Research Group (Canadá) y Australian Health Policy Collaboration (Australia) se han enfocado en el estudio de los niveles de actividad física y su relación con una vida saludable, identificando a la movilidad activa como un factor relevante para su incremento. Quizá un hito interesante en la materia ha sido la publicación, en el año 2020, por parte de la National Association of City Transportation Officials (NACTO), de su primer manual enfocado en niños: Designing Streets for Kids (National Association of City Transportation Officials [NACTO], 2020).

En otros casos, el objeto de estudio de los grupos de investigación es específicamente la movilidad activa a la escuela, como es el caso de Heart Foundation (Australia) y Promoting Fitness \& Health through Physical Activity (España). Este último, con su proyecto PACO: Pedalea y Anda al COle, posee tres objetivos principales: 1) estudiar los patrones de desplazamiento de 40.000 niños y adolescentes españoles en un período de 17 años; 2) diseñar y validar instrumentos para evaluar el modo de desplazamiento al colegio y promocionarlos entre adolescentes y padres; y 3) aplicar los instrumentos en colegios de Granada, Jaén y Valencia (PACO: Pedalea y Anda al Cole, 2017). De igual manera, en el año 2019, el Banco Interamericano de Desarrollo publicó a nivel regional el Toolkit: Herramienta para la implementación de caminos seguros a la escuela en la región de América Latina y el Caribe, el cual tiene como objetivo principal:

ser un recurso de referencia para guiar y animar a la planificación, diseño, implementación y seguimiento de los caminos escolares, mejorando así la seguridad vial, en los viajes hacia y desde las escuelas realizados por los niños en la región de América Latina y el Caribe (Ponce de León y Koinange, p. 8).

La literatura sobre el tema nos brinda una visión de los estudios que se han llevado a cabo hasta el momento. La revisión de Davison et al. (2008), realizada en función de estudios previos a 2007, encuentra que el origen y estatus socioeconómico intervienen en la movilidad activa, siendo los niños hispanos y africanos y los de nivel socioeconómico más bajo los que tienen más probabilidades de moverse activamente a las escuelas; así también el género, la cantidad de actividad física que realizan los padres y la ubicación de los hogares son los principales factores que influyen en la movilidad activa de los alumnos a las escuelas. Diez años después, la revisión de literatura realizada por Rothman et al. (2018) concluye que, si bien la distancia influye en la toma de decisiones para la movilidad de los niños a la escuela, tienen que considerarse aspectos como la situación socioeconómica, la diversidad racial y la discapacidad. Aranda-Balboa et al. (2019) revisan 27 artículos y organizan las barreras de los padres de niños en tres grandes categorías: personales, sociales y ambientales. Esta última es la más representativa, ya que dentro de ella se encuentran las subcategorías más reportadas: entorno construido, seguridad vial, distancia y seguridad personal. El soporte social (presencia/ausencia de niños o adultos en el camino) constituye una subcategoría de la categoría social y se encuentra en siguiente lugar. Por otro lado, Rojas Lopez y Wong (2017) encuentran que los factores que tienen asociación positiva con la movilidad activa de niños son 
principalmente la accesibilidad, la conectividad entre las rutas de la casa a la escuela y la percepción de los padres sobre estas rutas. Así también, en esta revisión de literatura se identificaron preferencias de acuerdo con el género, las niñas en general prefieren movilizarse acompañadas, mientras que los niños gustan de hacerlo de manera independiente y activa. La posibilidad que caminen o vayan en bicicleta a la escuela puede estar también determinada por el nivel de cohesión social, la conformación familiar (varios hermanos) y, sobre todo, por la tenencia de vehículo, el acceso a transporte público y la distancia. Finalmente, la revisión de Marzi et al. (2018) estudia la movilidad activa de los niños, pero enfocándose en cómo el sexo/género juega un papel importante en su movilidad, los resultados muestran que los niños son más independientes que las niñas.

Una característica que está presente en las revisiones de la literatura antes mencionadas es que la mayor parte de los estudios ha sido realizada principalmente en Norteamérica, Europa y Oceanía. Lo cual resulta inquietante dado que es justamente en los países en vías de desarrollo en donde optimizar las condiciones para la movilidad activa puede aportar a mejorar las condiciones de vida de su población. Esta situación ha sido comprobada, una vez más, en la revisión de literatura que se expone a continuación; en la cual, de todos los artículos revisados, apenas dos corresponden a Latinoamérica: el estudio realizado por Palma et al. (2019) en Valparaíso-Chile y el de HuertasDelgado et al. (2018) en Riobamba-Ecuador (Tabla 1).

Además de la brecha relacionada con las regiones en donde se han realizado los estudios, existen otras que deben ser salvadas por los investigadores en los próximos años. Por ejemplo, la mayor parte de las investigaciones ha sido elaborada con un diseño transversal, lo cual no permite esclarecer si las variables son causantes o resultantes de la movilidad activa (Davison et al. 2008; Marzi et al., 2018; Rothman et al., 2018). Así también, se evidencia que no existen definiciones y mediciones estandarizadas para la identificación y análisis de variables (Aranda-Balboa et al., 2019; Davison et al. 2008; Marzi et al., 2018).

Existen, además, algunas brechas cuya investigación podría coadyuvar para desarrollar estrategias de potenciación de la movilidad activa a los centros de estudio, tales como, por ejemplo: profundizar en la población de adolescentes (Aranda et al., 2019); en niños pequeños (la mayor parte de estudios se enfocan en niños de nueve a 12 años y muy pocos a un rango entre seis y nueve años) (Marzi et al., 2018); y en niños y jóvenes con discapacidad (Rothman et al., 2018). De acuerdo con Marzi et al. (2018) aún existe mucho por indagar en torno a la relación de género/sexo y movilidad activa a las escuelas.

Finalmente, es importante evidenciar que los estudios relacionados con la temática no cruzan la información con políticas públicas, por ejemplo, de transporte colectivo (Davison et al., 2008); o con temas como los beneficios económicos que la movilidad activa a la escuela conlleva, no únicamente a nivel del núcleo familiar, sino a diferentes escalas geográficas urbanas y en distintos ámbitos (salud física y mental, economía familiar, infraestructura, etc.) (Rojas Lopez \& Wong, 2017).

\section{Metodología}

\section{Formas de estudiar la movilidad activa de los escolares}

Los estudios seleccionados para la revisión de literatura expuesta evidencian enfoques tanto cuantitativos como cualitativos y mixtos. Las metodologías cuantitativas corresponden, por lo general, a la aplicación de encuestas y cuestionarios a padres y/o madres de familia y/o a los escolares (Huertas-Delgado et al., 2018; McDonald et al., 2014; Mendoza et al., 2014; Mitra et al., 2014; Palma et al., 2019; Sims, \& Bopp, 2019; Villanueva et al., 2014; Wilson et al., 2018). Los cuestionarios suelen incluir preguntas relacionadas con el género, la raza, nivel de ingresos, distancia a la escuela, de modo de identificar la relación que tienen con las formas de transporte y posibles barreras hacia la movilidad activa. Otros aplican entrevistas a profundidad (Banerjee et al., 2014; Joelsson, 2019). En el ámbito cualitativo, se utilizan también los grupos focales para entender aspectos relacionados con la percepción (Witten et al., 2013).

Otros estudios han implementado la cartografía y los mapas cognitivos para identificar lugares problemáticos, hitos que verifican los escolares en su camino al colegio, características de las rutas peatonales y ciclistas, entre otros factores (Halseth, \& Doddridge, 2000; Moran et al., 2018; Wridt, 2010). Algunos datos recolectados en mapas 
Tabla 1

Artículos seleccionados

\begin{tabular}{|c|c|c|}
\hline Autor(es) & Título del artículo & País abordado \\
\hline Aranda-Balboa et al. (2019) & Parental barriers to active transport to school: a systematic review & (revisión sistemática) \\
\hline Banerjee et al. (2014) & $\begin{array}{l}\text { Walking to School: The Experience of Children in Inner City Los Angeles and } \\
\text { Implications for Policy }\end{array}$ & Estados Unidos \\
\hline Bourke (2016) & $\begin{array}{l}\text { Children's experiences of their everyday walks through a complex urban } \\
\text { landscape of belonging }\end{array}$ & Irlanda \\
\hline Buliung et al. (2013) & $\begin{array}{l}\text { The "Path" Not Taken: Exploring Structural Differences in Mapped- Versus } \\
\text { Shortest-Network-Path School Travel Routes }\end{array}$ & Canadá \\
\hline Davison et al., 2008 & $\begin{array}{l}\text { Children's Active Commuting to School: Current Knowledge and Future } \\
\text { Directions }\end{array}$ & (revisión sistemática) \\
\hline Ferri-García et al. (2019) & $\begin{array}{l}\text { Data mining techniques to analyze the factors influencing active commuting } \\
\text { to school }\end{array}$ & España \\
\hline Halseth, \& Doddridge (2000) & Children's cognitive mapping: a potential tool for neighbourhood planning & Estados Unidos \\
\hline Huertas-Delgado et al. (2018) & $\begin{array}{l}\text { Parental perceived barriers to active commuting to school in Ecuadorian } \\
\text { youth }\end{array}$ & Ecuador \\
\hline Ikeda et al. (2019) & $\begin{array}{l}\text { Assessment of direct and indirect associations between children active } \\
\text { school travel and environmental, household and child factors using } \\
\text { structural equation modelling }\end{array}$ & Nueva Zelanda \\
\hline Joelsson (2019) & $\begin{array}{l}\text { 'So that we don't spoil them': understanding children's everyday mobility } \\
\text { through parents' affective practices }\end{array}$ & Suecia \\
\hline Marzi et al. (2018) & $\begin{array}{l}\text { Social and physical environmental correlates of independent mobility in } \\
\text { children: a systematic review taking sex/gender differences into account }\end{array}$ & (revisión sistemática) \\
\hline McDonald, \& Aalborg (2009) & Why Parents Drive Children to School & Estados Unidos \\
\hline McDonald et al. (2014) & Impact of the Safe Routes to School Program on Walking and Bicycling & Estados Unidos \\
\hline Mendoza et al. (2014) & $\begin{array}{l}\text { Predictors of Children's Active Commuting to School: An Observational } \\
\text { Evaluation in } 5 \text { U.S. Communities }\end{array}$ & Estados Unidos \\
\hline Mitra et al. (2014) & $\begin{array}{l}\text { Do parental perceptions of the neighbourhood environment influence } \\
\text { children's independent mobility? Evidence from Toronto, Canada }\end{array}$ & Canadá \\
\hline Moran et al. (2017) & $\begin{array}{l}\text { Getting to Know a Place: Built Environment Walkability and Children's } \\
\text { Spatial Representation of Their Home-School (h-s) Route }\end{array}$ & Israel \\
\hline Moran et al. (2018) & $\begin{array}{l}\text { Examining the role of trip destination and neighborhood attributes in } \\
\text { shaping environmental influences on children's route choice }\end{array}$ & Israel \\
\hline Palma et al. (2019) & $\begin{array}{l}\text { Perceived parental barriers towards active commuting to school in Chilean } \\
\text { children and adolescents of Valparaíso }\end{array}$ & Chile \\
\hline Rojas Lopez, \& Wong (2017) & Children's active trips to school: a review and analysis & (revisión sistemática) \\
\hline Rothman et al. (2018) & $\begin{array}{l}\text { The decline in active school transportation (AST): A systematic review of the } \\
\text { factors related to AST and changes in school transport over time in North } \\
\text { America }\end{array}$ & (revisión sistemática) \\
\hline Schützhofer et al. (2018) & $\begin{array}{l}\text { The development of traffic competences - do children need special } \\
\text { infrastructure to be safe in traffic? }\end{array}$ & (revisión sistemática) \\
\hline Sims, \& Bopp (2019) & $\begin{array}{l}\text { Using parental active travel behavior and beliefs to predict active travel to } \\
\text { school among children }\end{array}$ & Estados Unidos \\
\hline Stewart et al. (2012) & Common ground: Eight factors that influence walking and biking to school & (revisión sistemática) \\
\hline Villanueva et al. (2014) & $\begin{array}{l}\text { Does the walkability of neighbourhoods affect children's independent } \\
\text { mobility, independent of parental, socio-cultural and individual factors? }\end{array}$ & Australia \\
\hline Wilson et al. (2018) & $\begin{array}{l}\text { Children's perspectives on neighbourhood barriers and enablers to active } \\
\text { school travel: A participatory mapping study }\end{array}$ & Canadá \\
\hline Witten et al. (2013) & $\begin{array}{l}\text { New Zealand parents' understandings of the intergenerational decline in } \\
\text { children's independent outdoor play and active travel }\end{array}$ & Nueva Zelanda \\
\hline Wridt (2010) & $\begin{array}{l}\text { A qualitative GIS approach to mapping urban neighborhoods with children } \\
\text { to promote physical activity and child-friendly community planning }\end{array}$ & Estados Unidos \\
\hline
\end{tabular}


Tabla 2

Enfoques utilizados en los estudios revisados

\begin{tabular}{llll}
\hline & & Enfoque & \\
\hline Cuantitativo & Cualitativo & Mixto & Revisión de literatura \\
\hline Ferri-García et al. (2019) & Banerjee et al. (2014) & Buliung et al. (2013) & Aranda-Balboa et al. (2019) \\
\hline Huertas-Delgado et al. (2018) & Bourke (2016) & Ikeda et al. (2019) & Davison et al. (2008) \\
\hline McDonald, \& Aalborg (2009) & Halseth, \& Doddridge (2000) & Moran et al. (2017) & Marzi et al. (2018) \\
\hline McDonald et al. (2014) & Joelsson (2019) & Moran et al. (2018) & Rojas Lopez, \& Wong (2017) \\
\hline Mendoza et al. (2014) & Witten et al. (2013) & Rothman et al. (2018) \\
\hline Mitra et al. (2014) & Wridt (2010) & Schützhofer et al. (2018) \\
\hline Palma et al. (2019) & & Stewart et al. (2012) \\
\hline Sims, \& Bopp (2019) & & \\
\hline Villanueva et al. (2014) & & \\
\hline Wilson et al. (2018) & & \\
\hline
\end{tabular}

Tabla 3

Unidades de observación por estudio revisado

\begin{tabular}{lll}
\hline & \multicolumn{1}{c}{$\begin{array}{c}\text { Unidades de observación } \\
\text { (no incluye revisiones de literatura) }\end{array}$} & \\
\hline Escolares & Padres/Madres & Escolares + Padres/Madres \\
\hline Banerjee et al. (2014) & Huertas-Delgado et al. (2018) & Buliung et al. (2013) \\
\hline Bourke (2016) & Joelsson (2019) & Ikeda et al. (2019) \\
\hline Ferri-García et al. (2019) & McDonald, \& Aalborg (2009) & Mitra et al. (2014) \\
\hline Halseth, \& Doddridge (2000) & Mendoza et al. (2014) & Villanueva et al. (2014) \\
\hline McDonald et al. (2014) & Palma et al. (2019) & Wilson et al. (2018) \\
\hline Moran et al. (2017) & Sims, \& Bopp (2019) & \\
\hline Moran et al. (2018) & Witten et al. (2013) & \\
\hline Wridt (2010) & & \\
\hline
\end{tabular}


Tabla 4

Factores que inciden en la movilidad activa a la escuela

\begin{tabular}{|c|c|c|}
\hline \multicolumn{3}{|c|}{ Factores } \\
\hline Seguridad & Entorno construido & Distancia \\
\hline Aranda-Balboa et al. (2019) & Aranda-Balboa et al. (2019) & Aranda-Balboa et al. (2019) \\
\hline Banerjee et al. (2014) & Banerjee et al. (2014) & Buliung et al. (2013) \\
\hline Huertas-Delgado et al. (2018) & Bourke (2016) & Davison et al. (2008) \\
\hline Ikeda et al. (2019) & Buliung et al. (2013) & Ferri-García et al. (2019) \\
\hline Marzi et al. (2018) & Davison et al. (2008) & Huertas-Delgado et al. (2018) \\
\hline Palma et al. (2019) & Huertas-Delgado et al. (2018) & Ikeda et al. (2019) \\
\hline Rojas Lopez, \& Wong (2017) & Ikeda et al. (2019) & Moran et al. (2018) \\
\hline Schützhofer et al. (2018) & Marzi et al. (2018) & Palma et al. (2019) \\
\hline Stewart et al. (2012) & Moran et al. (2018) & Rojas Lopez, \& Wong (2017) \\
\hline Villanueva et al. (2014) & Palma et al. (2019) & Stewart et al. (2012) \\
\hline Wilson et al. (2018) & Rojas Lopez, \& Wong (2017) & Wilson et al. (2018) \\
\hline \multirow[t]{5}{*}{ Wridt (2010) } & Stewart et al. (2012) & \\
\hline & Villanueva et al. (2014) & \\
\hline & Wilson et al. (2018) & \\
\hline & Witten et al. (2013) & \\
\hline & Wridt (2010) & \\
\hline Entorno sociodemográfico & Conveniencia & Clima \\
\hline Banerjee et al. (2014) & Huertas-Delgado et al. (2018) & Davison et al. (2008) \\
\hline Bourke (2016) & Ikeda et al. (2019) & Ferri-García et al. (2019) \\
\hline Davison et al. (2008) & McDonald, \& Aalborg (2009) & Huertas-Delgado et al. (2018) \\
\hline Ferri-García et al. (2019) & Palma et al. (2019) & Palma et al. (2019) \\
\hline Huertas-Delgado et al. (2018) & Stewart et al. (2012) & Stewart et al. (2012) \\
\hline Ikeda et al. (2019) & Witten et al. (2013) & Wilson et al. (2018) \\
\hline \multicolumn{3}{|l|}{ Joelsson (2019) } \\
\hline \multicolumn{3}{|l|}{ Marzi et al. (2018) } \\
\hline \multicolumn{3}{|l|}{ Mitra et al. (2014) } \\
\hline \multicolumn{3}{|l|}{ Palma et al. (2019) } \\
\hline \multicolumn{3}{|l|}{ Rojas Lopez, \& Wong (2017) } \\
\hline \multicolumn{3}{|l|}{ Sims, \& Bopp (2019) } \\
\hline \multicolumn{3}{|l|}{ Stewart et al. (2012) } \\
\hline \multicolumn{3}{|l|}{ Villanueva et al. (2014) } \\
\hline \multicolumn{3}{|l|}{ Wilson et al. (2018) } \\
\hline Witten et al. (2013) & & \\
\hline
\end{tabular}


se conjugan con datos demográficos para identificar si existen relaciones entre ambos. Algunos autores cruzan la cartografía con entrevistas o encuestas (Buliung et al. 2013; Ikeda et al., 2019; Moran et al., 2017; Moran et al., 2018). En esta misma línea, Bourke (2017) aplica una metodología menos convencional, pero que, en estudios con altos presupuestos, puede ser viable: se basa en dar cámaras fotográficas a los escolares para que fotografíen elementos que se encuentran en su ruta a la escuela que consideren peligrosos, agradables o que les llamen la atención.

Así como se diferencian los enfoques en función de los instrumentos utilizados, también se distinguen con respecto a los sujetos de estudio. Por un lado, están los trabajos que aplican los instrumentos de investigación a los escolares y, por otro, los que lo hacen a los padres y/o madres. En algunos casos los instrumentos se aplican a escolares y padres y/o madres (Tabla 3). Las metodologías utilizadas con los escolares en ciertos casos son más dinámicas que las que se usan con los adultos; tal como el uso de las cámaras (Bourke, 2016) o los mapas cognitivos (Halseth, \& Doddridge, 2000; Wridt, 2010). Las herramientas que se aplican con los padres y/o madres son más directas; por lo general encuestas o entrevistas. Existen, sin embargo, ejemplos interesantes como el de Witten et al. (2013), en el cual se les solicitó que no se limitaran a hablar de sus percepciones actuales, sino que relataran sus propias experiencias de cuando eran pequeños.

Las barreras con respecto a la movilidad activa que distinguen los escolares suelen diferir de las percibidas por sus padres y/o madres (Wilson et al., 2018) y dependen del nivel de madurez e independencia de cada uno de ellos (Joelsson, 2019). El constructo mental que realiza un escolar sobre su entorno compromete cuatro de sus cinco sentidos y, por lo tanto, la experiencia, al moverse por un espacio, se hace más o menos placentera dependiendo de los elementos que percibe (Bourke, 2017). Sin embargo, se evidencia que las decisiones de movilidad de los menores están en su mayoría en manos de los padres y/o madres; por lo que teorías como la del comportamiento planificado, ТPB (por sus siglas en inglés: Theory of Planned of Behaviour), en la cual se considera que el comportamiento y el modo de transporte se puede predecir por intenciones, preferencias y restricciones de los sujetos (Havlíčková, \& Zámečník, 2020; Pang et al., 2018; Zaragoza et al., 2020) no puede aplicarse directamente a las decisiones de los escolares, quienes por su corta edad, aún no cuentan con la autonomía para decidir su modo de transporte.

\section{La seguridad como barrera en la movilidad activa de escolares}

Una de las principales barreras cuando se habla sobre la movilidad activa de los escolares es la seguridad vial y personal. Se entiende por seguridad vial aquella referida a la exposición a accidentes de tránsito, y por seguridad personal a los riesgos relacionados con crimen y violencia (Rojas López, \& Wong, 2017).

Existe una relación directa entre el nivel de movilidad activa y la percepción de seguridad (Ferri-García et al., 2019). Se ha demostrado que los padres y/o madres tienen una preocupación muy alta por la seguridad de sus hijos (Ferri-García et al., 2019; Huertas-Delgado et al., 2018; Ikeda et al., 2019; Palma et al., 2019; Wridt, 2010) y, por ende, tienden a ver el barrio de una manera más negativa que los escolares (Banerjee et al., 2014; Sims, \& Bopp, 2019). En función de la percepción que los padres y/o madres tengan de la ruta a la escuela, aumenta o disminuye la independencia que dan a sus hijos y, por tanto, su movilidad activa (Davison et al., 2008). Según Joelsson (2019), el contexto espacial, social y cultural da forma al pensamiento de los padres y/o madres y los convierte en "expertos en riesgos y en administradores de los riesgos" (p. 9), lo que causa que puedan limitar la movilidad de los escolares sobre la base de juicios de percepción; aparentemente, el protegerlos se puede entender como un acto afectivo. La comunicación, a través de las noticias exageradas de sucesos, tiene un rol importante en la intensificación del miedo de los padres y/o madres (Ikeda et al., 2019).

Como medidas que se pueden tomar al respecto, Ikeda et al. (2019) señalan que la percepción de seguridad vial puede mejorar al incorporar estructura peatonal y 
para ciclistas, como, por ejemplo: aceras, señalización y ciclovías. Así también, Wridt (2010) propone ciertas estrategias para que los escolares puedan manejar situaciones peligrosas, entre las cuales se mencionan: viajar acompañado de un adulto, llevar un número de teléfono de emergencia, conocer lugares del barrio donde puedan obtener ayuda. Otra sugerencia hace referencia a programas de educación para desarrollar las capacidades cognitivas de los escolares para, consecuentemente, aumentar la confianza de los padres y/o madres en permitir a sus hijos movilizarse de manera activa (Ikeda et al., 2019).

Con respecto a la capacidad cognitiva, de acuerdo con Wridt (2010), los escolares entre 10 y 11 años son capaces de ubicar y percibir posibles riesgos en su barrio al momento de caminar o ir en bicicleta a la escuela y, por lo tanto, evitar el peligro. Esto lo ratifican Schützhofer et al. (2018), quienes mencionan que, a los seis años en promedio, los escolares identifican el peligro muy tarde, cuando ya están dentro de una situación peligrosa; mientras que a los ocho pueden reconocer patrones de peligro, pero no evadirlos y, finalmente, entre los nueve y diez pueden desarrollar un comportamiento preventivo de forma consciente frente a este. Por esto, si bien la percepción de los padres y/o madres es importante para la toma de decisiones sobre la movilidad de sus hijos, las vivencias e impresiones de los escolares pueden ser determinantes al momento del planteamiento de políticas o estrategias. Por ejemplo, Wilson et al. (2018) identifican en su estudio que los estudiantes de las escuelas se sienten más seguros cuando se encuentran con policías de tránsito en las intersecciones.

En relación con la seguridad vial, la falta de infraestructura vial adecuada como ciclovías y aceras constituye un hecho determinante tanto para los escolares como para los padres y/o madres en sus decisiones de impedir o posponer la movilidad a pie o en bicicleta (Palma et al., 2019; Wilson et al., 2018; Wridt, 2010). Algo similar sucede con el caos vehicular que se produce en la zona de embarque y desembarque de los escolares en las horas de salida y entrada de las escuelas (Wridt, 2010). En la revisión de literatura de Aranda-Balboa et al. (2019) se señala que la mayor parte de estudios encuentran una asociación directa entre la percepción de inseguridad vial con la cantidad de tráfico y congestión, lo cual, según los autores, lleva a un círculo vicioso, ya que esa percepción de inseguridad hace que los padres prefieran trasladar a sus hijos en sus autos, generando a su vez más congestión.

Otro aspecto importante que resaltar es que el tema de la seguridad vial y personal está atravesado por la variable del género. La sobreprotección de los padres y/o madres hacia sus hijas es mayor que hacia los hijos según algunos autores (Davison et al., 2008; Stewart et al., 2012; Villanueva et al., 2014). En la revisión de literatura realizada por Davison et al. (2008) se menciona que la posibilidad de que los escolares varones se movilicen de forma activa es dos veces más alta en comparación con las mujeres. De igual manera, Villanueva et al. (2014) hacen una clara diferenciación entre las vivencias de varones y mujeres; los primeros son más independientes en su movilidad a la escuela y manejan un rango territorial más amplio, lo cual les permite estar más expuestos al ambiente del vecindario y, consecuentemente, manejar mejor sus riesgos, lo cual deriva en un círculo vicioso. Finalmente, la revisión de la literatura realizada por Marzi et al. (2018) encuentra que existen diferencias de movilidad por género, los resultados muestran que las niñas son menos independientes que los niños; en ciertos estudios, señalan los autores, se comprueba que se les permite estar afuera solo si son acompañadas por varones.

\section{Otros factores, más allá de la seguridad}

Además de la seguridad, la revisión de literatura realizada nos ha permitido evidenciar otros factores que inciden en las decisiones de movilidad activa de escolares; algunos de estos constituyen verdaderas barreras percibidas, principalmente por padres y/o madres, que conducen a la decisión de no permitir la movilidad activa en sus hijos y/o hijas.

\section{Entorno construido}

Varios estudios incluyen al entorno construido como un factor relevante para explicar la decisión de caminar o 
pedalear a la escuela (Aranda-Balboa et al.,2019; Rojas Lopez, \& Wong, 2017; Witten et al., 2013). En un estudio realizado en Israel, se verifica que la morfología urbana que caracteriza el lugar de residencia de un escolar determina la ruta escogida para caminar hacia la escuela. Como resultado, se identificó que los escolares procedentes de barrios centrales y densos escogieron rutas con una menor cantidad de intersecciones, mientras aquellos procedentes de barrios suburbanos priorizaron otra variable, la compacidad (Moran et al., 2018), es decir, la alta densidad poblacional. Este resultado indica una contradicción entre el tipo de barrio en el que se reside versus el barrio por el que prefieren caminar.

Por lo general, las rutas escogidas para caminar o ir en bicicleta a la escuela son entornos que cuentan con una alta densidad habitacional (Davison et al., 2008), baja densidad de intersecciones (Moran et al., 2018), trayectos no empinados (Davison et al., 2008), con altos niveles de caminabilidad (Aranda-Balboa et al., 2019; Davison et al., 2008) y, sobre todo, rutas directas y distancias cortas. Contrariamente a lo que podría suponerse, algunos estudios encuentran que, dentro del contexto construido, la diversidad de usos y la presencia de espacios abiertos recreacionales no inciden de manera determinante en esta decisión (Buliung et al., 2013; Davison et al., 2008). Por otro lado, para los escolares la imagen de los barrios sí constituye una barrera importante que inhibe la movilidad activa, por ejemplo, lugares en donde existen casas abandonadas o en mal estado (Bourke, 2017), basura y grafitis (Banerjee et al., 2014; Wilson et al., 2018) sí afectan su decisión. En cuanto al diseño urbano del barrio, la revisión de Marzi et al. (2018) evidencia que incide más en la movilidad activa de las niñas que en la de los niños. De la misma manera, en el estudio realizado por Banerjee et al. (2014), los escolares, al ser consultados sobre sus opiniones de cómo convertir a sus barrios en más caminables y seguros, la mayoría de las sugerencias estuvo ligada a los cambios que deben realizarse en el diseño urbano.

\section{Distancia}

La distancia hacia la escuela puede considerarse una de las barreras más importantes para la movilidad activa y existe una relación inversa entre ellas. Esto se ha demostrado tanto en estudios aplicados a escolares (Ferri-García et al., 2019; Ikeda et al., 2019; Moran et al., 2018; Rojas Lopez, \& Wong, 2017; Stewart et al., 2012; Wilson et al., 2018); como en estudios aplicados a padres y/o madres (Huertas-Delgado et al., 2018; Palma et al., 2019). Estos resultados llevan a poner a las relaciones de proximidad, como protagonistas de un futuro cambio en los modos de desplazamiento. Si bien la revisión de literatura de Marzi et al. (2018) considera que aún hay una brecha grande de conocimiento acerca del rango o distancia en la cual los niños se movilizan de manera independiente, autores como Ferri-García et al. (2019) y Rojas-Lopez y Wong (2017) coinciden en considerar que los escolares que viven a una distancia de hasta 1,6 kilómetros de la escuela tienen más probabilidad de caminar.

\section{Entorno sociodemográfico}

Según Bourke (2017), al momento de caminar por la ciudad o hacia la escuela, los escolares van construyendo un paisaje social, sensorial, pragmático e imaginativo. Por ello se puede afirmar que, mientras un escolar se movilice de una manera más activa, sus percepciones de la ciudad van a ser más detalladas y precisas. El autor menciona, además, como punto importante, que los escolares que caminan en su barrio interactúan con una cantidad alta de personas y, por ende, les ayuda a construir redes sociales. Al establecer estas redes, se genera un sentimiento de pertenencia, el que puede hacer más placentera la caminata. Concordante con esto, Ikeda et al. (2019) mencionan que la movilidad activa puede utilizarse para fortalecer los comportamientos sociales de los escolares.

No obstante, si bien están demostradas estas ventajas, existen barreras sociodemográficas que frenan la movilidad activa a las escuelas. Tal es el caso, por ejemplo, de aquellos fenómenos que impiden una apropiación del lugar o una interacción social sana entre escolares y adultos. Para Banerjee et al. (2014), las pandillas, drogas $\mathrm{y}$ violencia se hallan entre las principales barreras de este tipo, mientras que para Bourke (2017) es la presencia de personas fumando o bebiendo. 
En lo referente al nivel socioeconómico de los barrios, en Toronto-Canadá, Mitra et al. (2014) encontraron una clara diferenciación entre los barrios de ingresos altos y de ingresos bajos. En los primeros, la movilidad independiente de los escolares es menor que en la de barrios de ingresos bajos. Esto puede ir de la mano con lo planteado por Ikeda et al. (2019) quienes consideran que puede existir una presión social al ser percibido como un mal padre o un buen padre.

Asítambién, se evidencia una influencia de las características étnicas en las decisiones de movilidad (Ikeda et al., 2019; Witten et al., 2013). Por ejemplo, en un estudio realizado en Nueva Zelanda se encontró que las familias no permiten la movilidad independiente de sus hijos hacia barrios con características étnicas diferentes a la de ellos (Witten et al., 2013). Muchas veces estas emociones negativas se relacionan con procesos de estigmatización del barrio por parte de algunos padres y/o madres, sin tener necesariamente fundamentos (Joelsson, 2019).

\section{Conveniencia}

Otras razones que pueden ser identificadas como barreras que inhiben la movilidad activa se relacionan con situaciones de conveniencia de los padres y/o madres. Por ejemplo, en Nueva Zelanda, una de las razones por la que los padres y/o madres escogen la institución educativa es la reputación que esta tenga y no por la accesibilidad o cercanía; por ende, el modo de movilización a las escuelas depende de si la distancia permite o no la movilidad activa y/o de si los horarios de los padres y/o madres coinciden con los de los hijos para llevarlos (Ikeda et al., 2019). Así también, en ciertos casos, cuando la escuela queda cerca de la ruta al trabajo de los padres y/o madres, la movilidad independiente no es una opción que se considere (Huertas-Delgado et al., 2018; McDonald, \& Aalborg, 2009). En la revisión de literatura de Davison (2008), los padres y/o madres apoyan la movilidad activa cuando desplazarse en bicicleta o a pie no interfiere con sus horarios o tiempos de trabajo.

\section{Clima}

En algunos trabajos como el de Wilson et al. (2018), realizado en Ontario, se identifican elementos climáticos como la lluvia y la nieve como limitantes para la movilidad activa. Ferri-García et al. (2019), en su investigación en el sureste de España, añaden además la temperatura, el viento, las horas de alta radiación solar y la neblina. Estos estudios concluyen que todas estas condiciones meteorológicas son determinantes para los escolares al momento de decidirse por una movilización activa. Sin embargo, queda en evidencia que los resultados son contexto-dependientes, ya que autores como Buliung et al. (2013) y Davison et al. (2008) sostienen que el clima y la temperatura no siempre inciden en la decisión del modo de desplazamiento.

\section{Discusión y conclusiones}

Este texto ha dejado en evidencia que, si bien la seguridad vial y personal es una de las principales barreras que incide en las decisiones de movilidad, existen otras que inhiben la movilidad activa a la escuela, entre ellas: el entorno construido, la distancia, el entorno sociodemográfico, la conveniencia de padres y/o madres y el clima.

En lo que respecta a la seguridad vial se destacan como barreras la falta de infraestructura (ciclovías y aceras), y también la ausencia de control policial en cruces con alta presencia vehicular. Para efectos de la seguridad personal, resultan importantes limitantes la percepción de peligro en la zona y el género de los escolares, en este marco, las niñas se ven más limitadas en sus posibilidades de caminar o andar en bicicleta. Tanto en la seguridad vial como personal, el entorno construido juega un papel fundamental.

En este contexto, cada uno de los actores de la movilidad escolar, gobierno nacional y local, escuelas, padres y/o madres y escolares pueden trabajar en acciones tanto a corto como a largo plazo para incorporar mejoras significativas en la movilidad activa. Se esbozan a continuación algunas ideas:

\section{Gobierno nacional y local}

El gobierno, tanto a nivel nacional como local, debe garantizar las intervenciones en infraestructura de aceras, ciclovías, cruces peatonales, señalización, así 
como mejorar las condiciones de permeabilidad visual en fachadas, seguridad y vigilancia ciudadana, a la vez que reducir la velocidad autorizada de vehículos motorizados en las zonas escolares. Las soluciones a varias de las barreras que se han evidenciado en esta revisión de la literatura especializada no implican necesariamente grandes inversiones y son estrategias a corto plazo, a diferencia de los montos y tiempos que por lo general requiere la infraestructura para la movilidad motorizada. Tal como Banerjee et al. (2018) manifiestan, no siempre hace falta aumentar la cantidad e infraestructura de espacios públicos sino convertir en lugares seguros a los existentes. Así también, las instituciones públicas responsables de la movilidad urbana deben incrementar las actividades de educación vial a la ciudadanía para garantizar el respeto al peatón y al ciclista.

En el largo plazo, el gobierno, a través de diferentes instrumentos, deberá motivar que los escolares asistan a la escuela de su jurisdicción, evitando así las grandes distancias que, entre otros perjuicios, inhiben la movilidad activa, tal como han recomendado Morán et al. (2017) y Huertas -Delgado et al. (2018). Situación que ya se da en varios países, pero el éxito únicamente se logra cuando se ha equilibrado previamente el nivel y calidad de todas las escuelas de la ciudad. A nivel local, los planes de ordenamiento de las ciudades deberán contemplar siempre como estrategia la diversidad de usos de suelo en las rutas escolares, como parte del mejoramiento de la percepción de seguridad.

\section{Escuelas}

Las escuelas pueden aportar con la implementación de programas de motivación a la movilidad activa para padres y/o madres y escolares y, también, a través de su colaboración con las campañas de información y capacitación en el desarrollo de destrezas requeridas y en el manejo de riesgos potenciales. Autores como Palma et al. (2019) sugieren que estos temas deben ser parte de los currículos de las escuelas y de las actividades prácticas como las salidas de campo, ciclopaseos, etc. De igual forma, las escuelas deben ser partícipes de la mejora de las condiciones del barrio en el que se ubican y, sobre todo, tienen una responsabilidad directa de garantizar condiciones adecuadas para la movilidad activa en las zonas de embarque y desembarque, tal como sugieren Ikeda et al. (2019).

\section{Padres y/o madres}

Como se ha señalado, los padres y/o madres son quienes deciden fundamentalmente sobre la forma de desplazamiento de sus hijos y/o hijas, por ende, ellos también pueden contribuir con la mejora de las condiciones de la movilidad activa a la escuela. En primer lugar, a través de un conocimiento de los barrios y entornos, tanto de su zona de residencia como del establecimiento escolar, y una participación en las iniciativas de mejoramiento de estos. Esta participación debe incluir la interacción con los vecinos, como estrategia para incrementar la percepción de seguridad.

Dado que existe un consenso de que los padres y/o madres se sienten más tranquilos de que sus hijos y/o hijas se movilicen activamente cuando un adulto los acompaña, se pueden promover e incentivar programas para que los padres y/o madres se turnen para acompañar a un grupo de escolares, garantizando así una vigilancia permanente en el recorrido (McDonald, \& Aalborg, 2009; Mendoza et al., 2014).

De igual manera, los padres y/o madres tienen un rol en la educación de sus hijos y/o hijas para erradicar sentimientos de discriminación como la xenofobia o la aporofobia, que inhiben la cohesión social, profundizando la percepción de inseguridad. Los padres y/o madres pueden convertirse, además, en participantes activos de los programas a implementarse en las escuelas para potenciar la movilidad activa.

\section{Escolares}

Los escolares pueden aportar a potenciar la movilidad activa a través de su participación en los programas y capacitaciones organizados, ya sea por uno de los niveles de gobierno o por la escuela. Según autores como Ikeda et al. (2019), los niños deben ser tomados en cuenta en los procesos participativos para que las estrategias tengan el éxito esperado. Tal como se detalló en párrafos anteriores de este texto, a partir de los 9-10 
años, los niños y/o niñas pueden manejar mejor los riesgos, por lo que pueden convertirse en interlocutores de los beneficios experimentados en su salud física y en su rendimiento, así como en los beneficios colectivos respecto de la ciudad y del planeta relacionados con la contaminación y congestión.

En otras palabras, y coincidiendo con algunos autores como Huertas-Delgado et al. (2018), la educación, la participación y la comunicación son esenciales al momento de potenciar la movilidad activa.

La revisión de la literatura expuesta en este artículo ha reafirmado que los resultados son contexto-dependientes. Es evidente que, si se analizan las variables del clima, no será igual la movilidad activa a las escuelas en ciudades donde nieva versus aquellas de climas templados o si se toman en consideración las características sociodemográficas, frente a estas variables con seguridad existirán diferencias entre los países desarrollados y aquellos en vías de desarrollo. A modo de ejemplo, la Organización Mundial de la Salud considera a la obesidad como un problema grave en los países desarrollados y, por ende, la movilidad activa se convierte en una estrategia de ejercicio físico y salud; pero en los países en vías de desarrollo las problemáticas son otras, en ellos, la movilidad activa entra en juego como estrategia de mitigación de las desigualdades sociales. Por ello, el reto para la academia será justamente profundizar en la reflexión y debate acerca de esta temática en entornos incipientemente estudiados, tal es el caso de las ciudades de países en vías de desarrollo en los cuales las tasas de motorización han crecido sustancialmente en las últimas décadas (Dimitriou, 2011) y que, tal como se expuso en la Tabla 1, han sido poco investigados.

Así también, será importante la comparación de resultados entre ciudades por condiciones geográficas, sociales, económicas, morfológicas, entre otras. Para ello, será necesario que tal como señalan Aranda-Balboa et al. (2019), Davison et al. (2008) o Marzi et al. (2018) se homogenice la terminología e instrumentos para una adecuada comunicación entre investigadores, expertos y tomadores de decisiones. En este sentido, la creación de redes académicas será prioritaria. Se empiezan a evidenciar esfuerzos como la creación de la Red Iberoamericana de Investigadores en Desplazamiento Activo, Salud y Sostenibilidad, RIIDASS, conformada por 20 universidades de ocho países iberoamericanos ${ }^{1}$.

Además de las brechas regionales que deben cubrirse en la investigación sobre movilidad activa hacia las escuelas, se encuentran aquellos vacíos relacionados con otras poblaciones que también se desplazan cotidianamente en esta dirección, pero que han sido poco estudiados: adolescentes y discapacitados. De igual manera, deberán atenderse aquellas brechas de conocimiento acerca de la relación entre movilidad activa y género.

Será interesante también que las futuras investigaciones encuentren las relaciones entre la movilidad activa a las escuelas y la política pública de movilidad planteada por los países y ciudades. Por ejemplo, la política pública relacionada con el transporte público y el uso del vehículo privado tendrá incidencia sobre las decisiones de movilidad activa. Estos análisis deberán contemplar cómo se ha pasado de un nivel político a uno operativo, visibilizado en montos de inversión, normativa, proyectos, entre otros. Para ello, será necesario contar con estudios longitudinales (ya que la mayoría han sido transversales), que permitan comprender los cambios a lo largo del tiempo, y las causalidades o consecuencias del fenómeno.

La investigación en la temática deberá profundizarse aún más en las actuales circunstancias, dentro de la crisis sanitaria provocada por el COVID-19, ya que los trabajos expuestos en este texto son previos a la pandemia mundial. Entre las reflexiones que surgen con respecto a la movilidad activa en relación con la pandemia están: por un lado, el distanciamiento físico necesario para evitar el contagio, el cual obliga a pensar en intervenciones y transformaciones de las infraestructuras viales, como por ejemplo el ensanchamiento de aceras que permita el distanciamiento. Por otro lado, la pandemia ha conducido a reforzar las ventajas de la proximidad, concepto que

1 Ver más información en https://riidass.com/ 
desde hace algunas décadas ya se lo debatía como una posible estrategia de mitigación de los impactos provocados por la ciudad dispersa y orientada al automóvil privado. La proximidad, con las medidas de confinamiento y restricción de circulación vehicular, dictadas en diversos países para controlar la pandemia, permitió poner en evidencia sus ventajas y su potencial como catalizador de cambios hacia una movilidad no motorizada.

Se espera que trabajos como este aporten en la reflexión y planteamiento de políticas públicas, tomando en cuenta que, si se diseña el espacio público para incentivar la movilidad activa en los escolares, se está construyendo una ciudad más inclusiva para todos, ya que las estrategias para garantizar adecuadas condiciones para ellos indudablemente funcionan para personas de la tercera edad, con movilidad reducida y también para las encargadas de las labores de cuidado. ${ }^{45}$

\section{Referencias bibliográficas}

Aranda-Balboa, M. J., Huertas-Delgado, F. J., HerradorColmenero, M., Cardon, G., \& Chillón, P. (2020). Parental barriers to active transport to school: A systematic review. International Journal of Public Health, 65(1), 87-98. https://doi.org/10.1007/s00038-019-01313-1

Banerjee, T., Uhm, J., \& Bahl, D. (2014). Walking to School: The Experience of Children in Inner City Los Angeles and Implications for Policy. Journal of Planning Education and Research, 34(2), 123-140. https://doi. org/10.1177/0739456X14522494

Bourke, J. (2017). Children's experiences of their everyday walks through a complex urban landscape of belonging. Children's Geographies, 15(1), 93-106_https://doi.org/ 10.1080/14733285.2016.1192582

Buliung, R. N., Larsen, K., Faulkner, G. E. J., \& Stone, M. R. (2013). The "Path" Not Taken: Exploring Structural Differences in Mapped-Versus Shortest-Network-Path School Travel Routes. American Journal of Public Health, 103(9), 15891596. https://doi.org/10.2105/ajph.2012.301172
Davison, K. K., Werder, J. L., \& Lawson, C. T. (2008). Children's active commuting to school: current knowledge and future directions. Preventing Chronic Disease, 5(3), A100. https://www.ncbi.nlm.nih.gov/pubmed/18558018

Dimitriou, H. T. (2011). Transport and city development: understanding the fundamentals. En H. Dimitriou, \& R. Gakenheimer (Eds.), Urban Transport in the Developing World (pp. 8-39). Edward Elgar Publishing. https://doi. org/10.4337/9781849808392.00010

Ferri-García, R., Fernández-Luna, J. M., Rodríguez-Lopez, C., \& Chillón, P. (2019). Data mining techniques to analyze the factors influencing active commuting to school. International Journal of Sustainable Transportation, 14(4), 308-323. https://doi.org/10.1080/15568318.20 18.1547465

Goodman, A., Rojas, I. F., Woodcock, J., Aldred, R., Berkoff, N., Morgan, M., Abbas, A., \& Lovelace, R. (2019). Scenarios of cycling to school in England, and associated health and carbon impacts: Application of the 'Propensity to Cycle Tool'. Journal of Transport \& Health, 12, 263-278. https://doi.org/10.1016/j.jth.2019.01.008

Halseth, G., \& Doddridge, J. (2000). Children's Cognitive Mapping: A Potential Tool for Neighbourhood Planning. Environment and Planning. B, Planning \& Design, 27(4), 565-582. https://doi.org/10.1068/b2666

Havlíčková, D., \& Zámečník, P. (2020). Considering Habit in Research on Travel Mode Choice: A Literature Review with a Two-Level Methodology. Transactions on Transport Sciences, 11(1), 18-32. https://doi.org/10.5507/ tots.2020.004

Huertas-Delgado, F. J., Chillón, P., Barranco-Ruiz, Y., Herrador-Colmenero, M., Rodríguez-Rodríguez, F., \& Villa-González, E. (2018). Parental perceived barriers to active commuting to school in Ecuadorian youth. Journal of Transport \& Health, 10, 290-296. https://doi. org/10.1016/j.jth.2018.05.102 
Ikeda, E., Hinckson, E., Witten, K., \& Smith, M. (2019). Assessment of direct and indirect associations between children active school travel and environmental, household and child factors using structural equation modelling. The International Journal of Behavioral Nutrition and Physical Activity, 16(1), 32. https://doi.org/10.1186/ s12966-019-0794-5

Joelsson, T. (2019). “So that we don”tspoil them': understanding children's everyday mobility through parents' affective practices. Children's Geographies, 17(5), 591-602.https:// doi.org/10.1080/14733285.2019.1582752

Jones, R. A., Blackburn, N. E., Woods, C., Byrne, M., Van Nassau, F., \& Tully, M. A. (2019). Interventions promoting active transport to school in children: A systematic review and meta-analysis. Preventive Medicine, 123, 232-241. https:// doi.org/10.1016/j.ypmed.2019.03.030

Marzi, I., Demetriou, Y., \& Reimers, A. K. (2018). Social and physical environmental correlates of independent mobility in children: a systematic review taking sex/ gender differences into account. International Journal of Health Geographics, 17(1), 24. https://doi.org/10.1186/ s12942-018-0145-9

McDonald, N. C., \& Aalborg, A. E. (2009). Why Parents Drive Children to School: Implications for Safe Routes to School Programs. Journal of the American Planning Association. American Planning Association, 75(3), 331-342. https:// doi.org/10.1080/01944360902988794

McDonald, N. C., Steiner, R. L., Lee, C., Smith, T. R., Zhu, X., \& Yang, Y. (2014). Impact of the Safe Routes to School Program on Walking and Bicycling. Journal of the American Planning Association, 80(2), 153-167. https://doi.org/1 $\underline{0.1080 / 01944363.2014 .956654}$

Mendoza, J. A., Cowan, D., \& Liu, Y. (2014). Predictors of Children's Active Commuting to School: An Observational Evaluation in 5 U.S. Communities. Journal of Physical Activity and Health, 11(4), 729-733. https://doi. org/10.1123/jpah.2012-0322
Mitra, R. (2013). Independent Mobility and Mode Choice for School Transportation: A Review and Framework for Future Research. Transport Reviews, 33(1), 21-43. https:// doi.org/10.1080/01441647.2012.743490

Mitra, R., Faulkner, G. E., Buliung, R. N., \& Stone, M. R. (2014). Do parental perceptions of the neighbourhood environment influence children's independent mobility? Evidence from Toronto, Canada. Urban Studies, 51(16), 3401-3419. https://doi.org/10.1177/0042098013519140

Mokkink, L. B., Terwee, C. B., Gibbons, E., Stratford, P. W., Alonso, J., Patrick, D. L., Knol, D. L., Bouter, L. M., \& de Vet, H. C. W. (2010). Inter-rater agreement and reliability of the COSMIN (Consensus-based Standards for the selection of health status Measurement Instruments) checklist. BMC Medical Research Methodology, 10, 82. https://doi.org/10.1186/1471-2288-10-82

Moran, M., Eizenberg, E., \& Plaut, P. (2017). Getting to Know a Place: Built Environment Walkability and Children's Spatial Representation of Their Home-School (h-s) Route. International Journal of Environmental Research and Public Health, 14(6), 607. https://doi.org/10.3390/ ijerph14060607

Moran, M. R., Rodríguez, D. A., \& Corburn, J. (2018). Examining the role of trip destination and neighborhood attributes in shaping environmental influences on children's route choice. Transportation Research Part D: Transport and Environment, 65, 63-81. https://doi.org/10.1016/j. trd.2018.08.001

National Association of City Transportation Officials. (2020). Designing Streets for Kids. Island Press.

Palma, X., Chillón, P., Rodríguez-Rodríguez, F., Barranco-Ruiz, Y., \& Huertas-Delgado, F. J. (2019). Perceived parental barriers towards active commuting to school in Chilean children and adolescents of Valparaíso. International Journal of Sustainable Transportation, 14(7), 525-532. https://doi.org/10.1080/15568318.2019.1578840 
Pang, B., Rundle-Thiele, S., \& Kubacki, K. (2018). Can the theory of planned behaviour explain walking to and from school among Australian children? A social marketing formative research study. International Journal of Nonprofit and Voluntary Sector Marketing, 23(2), e1599. https://doi. org/10.1002/nvsm.1599

Ponce de León, M. y Koinange, C. (2019). Toolkit: Herramienta para la implementación de Caminos Seguros a la Escuela en la región de América Latina y el Caribe. Banco Interamericano de Desarrollo. https://publications. iadb.org/es/herramienta-para-la-implementacionde-caminos-seguros-la-escuela-en-la-region-deamerica-latina-y

PACO: Pedalea y Anda al COlegio (23 de enero de 2017). Promoting Fitness \& Health through Physical Activity. http://profith.ugr.es/pages/tablon/*/noticias/pacopedalea-y-anda-al-colegio-2\#.XvUKj21KjDc

Rojas Lopez, M. C., \& Wong, Y. D. (2017). Children's active trips to school: a review and analysis. International Journal of Urban Sustainable Development, 9(1), 79-95. https:// doi.org/10.1080/19463138.2016.1264405

Rothman, L., Macpherson, A. K., Ross, T., \& Buliung, R. N. (2018). The decline in active school transportation (AST): A systematic review of the factors related to AST and changes in school transport over time in North America. Preventive Medicine, 111, 314-322. https://doi. org/10.1016/j.ypmed.2017.11.018

Schützhofer, B., Rauch, J., \& Stark, J. (2018). The development of traffic competences - do children need special infrastructure to be safe in traffic? Transactions on Transport Sciences, 9(2), 3-17. https://doi.org/10.5507/ tots.2018.011
Sims, D., \& Bopp, M. (2019). Using parental active travel behavior and beliefs to predict active travel to school among children. International Journal of Sustainable Transportation, 14(20), 343-348. https://doi.org/10.10 80/15568318.2018.1558469

Smith, L., Norgate, S. H., Cherrett, T., Davies, N., Winstanley, C., \& Harding, M. (2015). Walking school buses as a form of active transportation for children-a review of the evidence. Journal of School Health, 85(3), 197-210. https://doi.org/10.1111/josh.12239

Stewart, O., Moudon, A. V., \& Claybrooke, C. (2012). Common ground: Eight factors that influence walking and biking to school. Transport Policy, 24, 240-248. https://doi. org/10.1016/j.tranpol.2012.06.016

Teixeira, J. F., Silva, C., \& Neves, J. V. (2019). School mobility management case study: German School of Oporto (Deutsche Schule zu Porto). Case Studies on TransportPolicy, 7(1), 13-21. https://doi.org/10.1016/j.cstp.2018.11.002

Villa-González, E., Barranco-Ruiz, Y., Evenson, K. R., \& Chillón, P. (2018). Systematic review of interventions for promoting active school transport. Preventive Medicine, 111, 115134. https://doi.org/10.1016/j.ypmed.2018.02.010

Villanueva, K., Giles-Corti, B., Bulsara, M., Trapp, G., Timperio, A., McCormack, G., \& Van Niel, K. (2014). Does the walkability of neighbourhoods affect children's independent mobility, independent of parental, socio-cultural and individual factors? Children's Geographies, 12(4), 393-411. https:// doi.org/10.1080/14733285.2013.812311

Wilson, K., Clark, A. F., \& Gilliland, J. A. (2018). Understanding child and parent perceptions of barriers influencing children's active school travel. BMC Public Health, 18(1), 1053. https://doi.org/10.1186/s12889-018-5874-y 
Witten, K., Kearns, R., Carroll, P., Asiasiga, L., \& Tava'e, N. (2013). New Zealand parents' understandings of the intergenerational decline in children's independent outdoor play and active travel. Children's Geographies, 11(2), 215-229. https://doi.org/10.1080/14733285.20 13.779839

Wridt, P. (2010). A Qualitative GIS Approach to Mapping Urban Neighborhoods with Children to Promote Physical Activity and Child-Friendly Community Planning. Environment and Planning. B: Planning \& Design, 37(1), 129-147. https://doi.org/10.1068/b35002

Zaragoza, J., Corral, A., Ikeda, E., García-Bengoechea, E., \&Aibar, A. (2020). Assessment of psychological, social cognitive and perceived environmental influences on children's active transport to school. Journal of Transport \& Health, 16, 100839.https://doi.org/10.1016/j.jth.2020.100839 